UCRL-esses

Preprint

UCRL--95366

DE87 009573

\title{
Utilization of Optical In rage Data from the Advanced Test Accelerator (ATA)
}
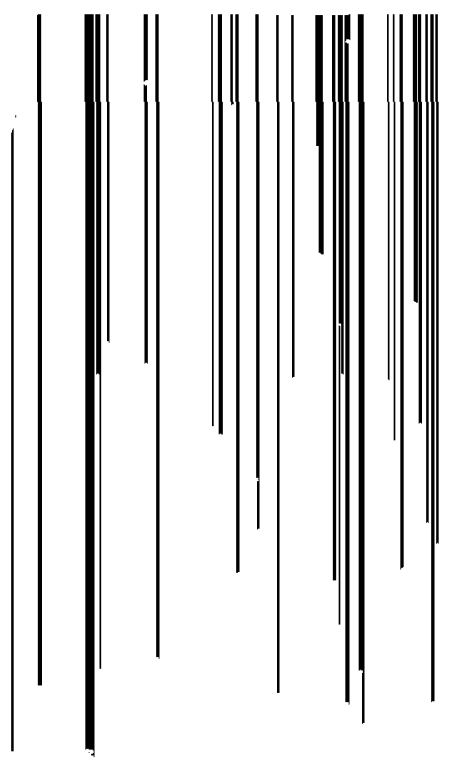

F. W. Chambers, J. S. Kallman, M. E. Slominski, Y. P. Chong, D. Donne ly, and J. P. Comish

Prepared ff - submittel to SPIE 1987 ' chnical Symposium Southeast on Optics, Electro-Optics, and Sensors

Orlando, Florida

May 17-22, 1987

\section{Beam}




\section{DISCLAIMER}

This report wiss prepared as an account of work sponsored by an agency of the United States Government. Neither the Unized States Goyernment nor any ugency therecl, nor uny of theip employes, makes asy watranty, express or implied, of assumes any legal liability or responsibility for the jccuracy, compieteness, or usefuiness of any information, apparatus, product, or process diselosed. of represents that its usu would not infringe privately owned rights. Reference herein to any specilic commercisl product, process, or service by trade name, stademark, mönufacturer, or utherwise does not necessarily constitute or imply its endorsement, recommendation, of favoriag by the United Stales Government or any agency thereof. The views and opinions of authors expressed herein do not necessarily state or renect those of the United States Government or any agency thereul. 
Utilization of optical image data fron the Advanced Test Acceierator (ATA)

F. H. Chambers, J. S. Kallman. H. E. Slominski, Y. P. Chong, D. Donnelly, and J. P. Cornish

Lawrence Livermore Mational Laboratory, University of California

P. D. Box 808, L626, Livermore, Ealifornia 94550

\section{Abstract}

Extensive use is made of optical diagnostics to obtain Information on the 50-HeV, 10-kA, 10-ns pulsedelectron beam produced by the Advanced Test Accelerator (ATA). I Light is generated by the beam striking a foll inserted in the beamline or through excitation of the gas when the beamline is filled with air. The enitted light is collected and digitized. Two-dimensional images are recorded by either a gated fraaing camera or a streak camera, Extraction of relevant beam parameters such as current density, current, and beam slze requires an understanding of the physics of the light-generation mechanisn and an ability to handle and properly exploit a large digital database of image data. We will present a brief overuiew of the present understanding of the light-generation mechanisms in foil and gas, with emphasis on experinental observations and trends. We will review our data management and analysis techniques and indicate successful approaches for extracting beam parameters.

\section{Introduction: ATA and optical dtagnostics}

The Advanced Test Accelerator (ATA), as described in a companion paper by Chong, et al. 1 is an induction linac that produces electron-beam pulses of $10 \mathrm{kA}$ at $50 \mathrm{MeV}$ for $70 \mathrm{~ns}$ with a pulse-repetition frequency of $1 \mathrm{~Hz}$. The machine is remoteiy siled at the Lawrence Livermore Natlonal Laboratory's Site 300, located approximately 50 miles east of San Francisco. The ATA was funded by the Defense Advanced Research Projects Agency (DARPA) and is being used to study electron-beam transport under a variety of conditions.

Uptical diagnostics are used extensively for studying the electron beam in ATA. Light is generated by the beam striking a target foil or passing through gas in the beamline. This light is transmitted to varlous detectors well removed from the beamline by optical lines of sight (LOS). The optical data allow experimenters to oetermine the electron beam position, size, profile, and intensity.

The time stales of interest for ATA LOS diagnostics are typically several nanoseconds. If optical systems can be gated to this speed, the gates can be moved on a pulse-to-pulse basis. Typical LoSs will view a field of $15-30 \mathrm{~cm}$; the desired spatial resolution is 1 mor less. The desired dynamic range on intensity for a single LOS is $100: 1$.

Opticai diagnostics are used in two very distinct modes. The first mode is as a rapid qualitative bean diagnostic for tuning the accelerator (1.t.. varying magnetic fields, gas proflles, etc.). In this mode, the data must he displayed in a convenient form (usually an inage) for the selected diagnostic. The data are properly labeled at the $\mathrm{l}-\mathrm{Hz}$ repetition rate, and displayed at many locations throughout the control room and several diagnostic areas. A second complementary function for the optical diagnostics is to make quantitative measurements of beam parameters. Light measurements require a detalled understanding of the calibrations throughout the LOS and the detector. Moreover, to determire electron-beam parameters, a good understanding of the physics of 1 ight generation in the foif or gas is necessary. In this case, rapid data analysis on a $1-5$ time scale is not required, but sufficlent data must be recorded to permit subsequent analysis of the inage. The ATA optical diagnostic system has been designed to perform in both of these mortes.

\section{The ATA environment}

The ATA is located in a subterranean hall and is approximately $75 n$ in length. Between 25 and 40 m of transport are required beyond the accelerator to bring the beam into one of the three experinental halis that are located in horizontal underground tunnels. Typical experiments are 20 m or so in length. Over the 100 to 140 m of che accelerator, transport, and experiment, about 10 LOSs are deployed; at any given time only one or several of these Loss are in use.

The ATS presents a hostile environment in several respects. The electron bean produces copious radiation in the tunnel on each shot.2 Upwards of 2000 rad may be produced on each pulse. This radiation is in the form of high-energy $x$ rays and neutrons. The radiation is concentrated in the forward direction relative to the electron beam propagation and is often localized in a region of beam spill or at the dump where the beam is intentionally spilled. This radiation environment produces spurious signals on all signal-carrying coaxial cables in the tunnel. The radiation precludes the tunnel fron being occupied by personnel during a shot. Activation of beamline components during an evening's run may render the tunnel unsafe for entry until the following morning. Hence, all diagnostics in the tunnel must be static or remotely controlled. The radiation environment can also preclude introduction of optical components into the tunnel. The electron beam and the accelerator pulse-power systems produce an electromagnetic pulse (EMP) each time the accelerator is fired. This EMP can wreak havoc with control and safety systems and contributes tens of 
millivolts to any electronic signal measured in the tunnel. The radiation and EMP in the tunnel preclude the use of solid-state electronic companents uniess they can be properly shlelded.

The ATA tunnel presents a hostile entironment in several more subtle ways. Access to the various experimental alcoves is quite limited, as the tunnels are less than $3.5 \mathrm{~m}$ wide at points. The beamine, yacuum lines, gas lines, water lines, and cable trays all compeie for linited space. The optical Loss, which require clear paths but do not reserve these paths with pipes or housings, are often violated by inadvertent piacenent of hardware. Because the ATA is a very dynamic facility with continuing beamitne construction. dust is continually deposited on all optical components, changing transmission characteristics. Maintenance of alignnent is difficult, as mirrors and mounts are subject to bumping by personnel and forklifts.

In this section, we have enumerated several aspects of the hostile environment presented for optical diagnostics at the ATA. In the next section. we will present the solutions we have found to achieve the reasurement capabilities outlined in the introductory section, given this environnent.

\section{The ATA optical diagnostics systems}

The ATA optical diagnostic system is lllustrated in Fig. 1, whtch shows the optical path for a typical LoS. The light signal is converted to a composite video signal by a camera in the rf enclosure. The composite video signal is then manipulated, recorded on videocassette recorders (VCR), broadcast to remote viewing monitors, and passed on to digitizing systems. The digitized data are then analyzed in several locations with complementary capabilities. In the remainder of this paper, we discuss the rationale for the vartous components in Fig. 1.

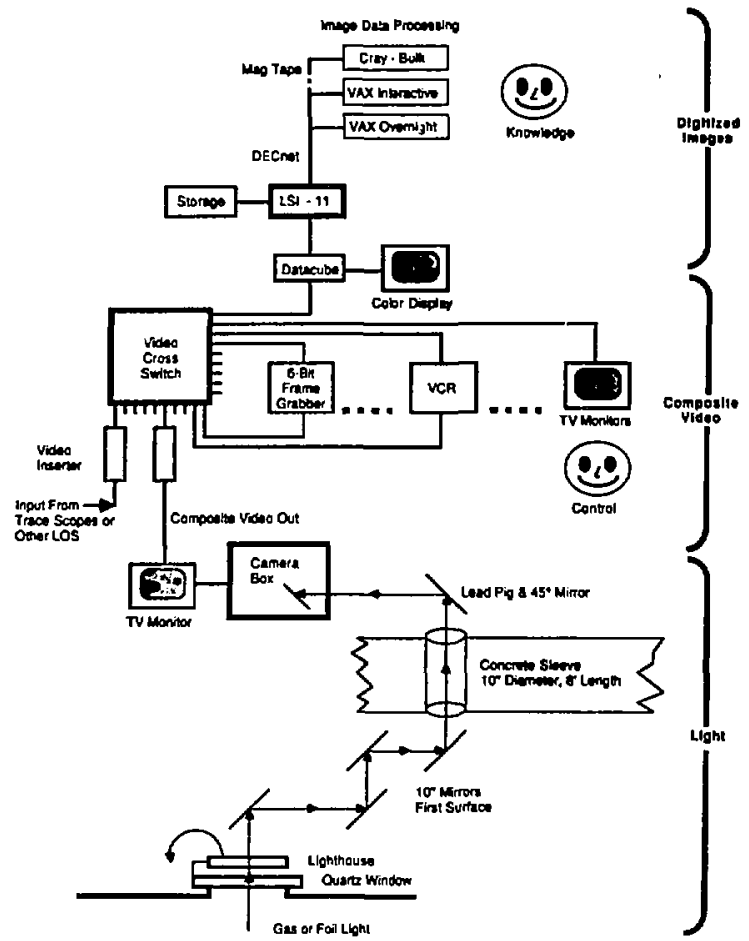

Figure 1. Schematic diagram of the ATA opt1cal diagnostic system. 
The optical traln-layout

ATA LOSS are engineered so that all optical detectors and optical components other than front surface mirrors are located outside the ATA tunnel. Light originates at a fixed foil in the beamline, an insertible foil employing a general-purpose probe (GPP). or as electron-beam-generated gas light. Light is transmitted through quartz windows on the beamline and then reflected with mirrors to penetrations or sleeves in the cancrete above the tunnel. These pentrations, efther ariginally cast in the concrete or later drilled through, have a nominal io-in. diameter and typically limit the effective diameter of the optical system to 8 in. From the source to the detector, the optical path length of a LOS is typically $10-15 \mathrm{~m}$. Thus. if the jight source is isotropic and the detector has an B-in.-diam aperture at $10 \mathrm{~m}$, only $2.6 \times 10^{-5}$ of the 1 ight reaches the detector. This low collection efficlency is adequate for very narrow electron beams at high currents. At lower currents, for diffuse beams, or for short time gates, this callection efficiency is too low and poor signal-to-notse levels are observed.

One way to increase light collection is to place an optical periscope in the concrete sleeve, thus reducing the distance from the source to the first optical. 'ement by several meters. In the past. periscopes have been successfully deployed at ATA. At one time, a lens was placed approximately 6 in. fron the beamiline to obtain a wide-angle view of the beam in the pipe. This diagnostic was extremely popular and useful, but the object lens (a standard 0lympus camera lens) darkened with $1 \mathrm{n} 1 / 2$ to $2 \mathrm{~h}$ of run time: the lens had to be replaced each day. At present, the low light levels encountered with the long LOSs are partially compensated by the use of high-gain (e.g., dual microchannel plate) cameras. In cases where the beam-generated light is predicted to come out in a narrow, highly directed cone (e.g.. optical transition radiation from a foll), the very narrow acceptance of the long los makes locating and measuring the radiation pattern quite difficult. For detecting light in narrow cone angles, alignment is done very carefully; however, beam shot-to-shot variations can often frustrate the careful alignment. At present. we are employing microchannel plate photomultiplers in the tunnel with massive amounts of shielding for $x$-ray medsurements. In the future, we wlll deploy a shielded camera in the tunnel to increase light-collection efficiency.

\section{The optical train-alignment, calibration, and documentation}

Because we are typically mintaining up to ten LOSs in parallel, the alignaent, calibration, and documentation must be carefuliy maintained. Typical Loss may have five mirrors mounted in the tunnel. usually on stands and mirror mounts. The alignment is performed with an alignment helfum-neon laser iocated in the rf enclosure. Calibration is achleved by fmaging a $1-\mathrm{cm}^{2}$ quadrille pattern placed on the entrance port window. Special fixtures called "lighthouses" are placed at each beamline view port; these lighthouses can be manually flipped in front of the view port. The fixtures cortain flluminated quadrilles that show the orientation and the label for the LOS. By viewing this lighthouse pattern, we can verify the LOS alignment each day. Given that the primary cause of loss of alignment is mirrors being bumped, all nirrors in the tunnel are labeled with their LOS number and a sequence number within the LOS. Signs in the tunnel tell workers how to report moved mirrors and strongly encourage them to do so. To document each LOS, we have developed a small computer program running on an IBH-PC. This progran takes the size calibration, center location, and orientation of the image produced by the LOS and produces a sample inage fron the LoS for several bean diameters, as seen in Fig. 2. A line drawing of the LoS is produced and the input LOS parameters are documented. This one-page LOS description is very useful in making quantitative estinates from observed images.

The LOS documented in Fig. 2 is actually a dual LOS in which two images are combined with a partially silvered mirror and projected onto one camera. The LOS views two fixed folls in the beamiline. The images fron the two optical LOSs are aligned to fall to the left and right in the inage plane. The ovals on the simulated image are for diameters of 2,4 , and $6 \mathrm{~cm}$; the images are oval because the foils are being viewed at $45^{\circ}$ as shown in the line drawing of the LDS.

\section{Data capture}

The light fram the LOS is captured by one of three transducers. 1 gated framing canera is typically used to capture an image with two spatial dimensions and a time gate of 3 ns or longer. A streak camera can be deployed to obtain data in time and one spatial dimension. In this case, the limit on temporal resolution has been low light levels rather than the streak sweep speed. A photomultiplier is sometimes used to obtain the time history only of the light. In this case, the signal is transmitted to the trace digitizing system (1.e.. oscilloscopes) to be recorded and sisplayed.

\section{Data display and recording - yideo}

To provide rapid data display in a convenient form for eight channels of video data, each canera signal is piped through a video inserter to a video cross switch, as shown in Fig. 1. The video inserter is used to superimpose a tine, date, and diagnostic identifier onto the image. This composite image can then be selected using the video cross switch and sent to one or more monitors located throughout the ATA complex. Banks of monitors are located in the control room, screen room, and diagnostic ounker to permit experimentalists to monitor the machine operation and experimental results. A special set of six monitors and a color monitor are located in the ATA conference roon to accomodate isiting observers. The composite video output from our oscilloscopes is also labeled and plped arourg the site using this system. (One drawback is that certain 

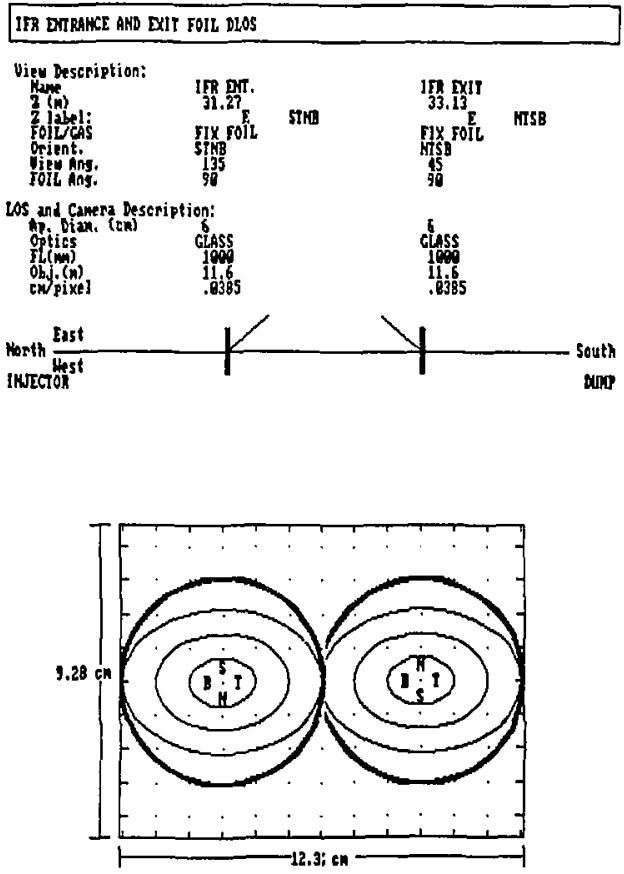

COWEms:

Figure 2. Documentation for a single LOS showing the location, orientation, and size scale for a particular LOS.

Japanese streak cameras and European oscilloscopes do not follow the Anerican standard for composite videc output.) Our system has proven extremely flexible and useful for the operational phase of the experiments.

Associated with this system are six VCRs used to record any six selected video channels (either insges or views of oscllloscope traces). The VCR records lack resolution and dymanic range, but they permit the convenient storage of an incredible bulk of data in a small volume at a low cost. Th1s capability is particularly valuable for documenting machine performance and reconstructing enomalous events that may occur on shot: that are not belng captured by the digitizing system. Two drabacks of the VCR systen are that cataloging the tapes is very tedious and accessing the images is not straightforward.

\section{Data display and recording - digital}

Two channels of image data tan be directed to LSI-1] conputers and captured on "datacubes" on thich the digitizer resolution is 240 pixels by 320 pixels with an 8 -bit dynamic range. The digitized image is displayed at $1 \mathrm{~Hz}$. A selected image can be "grabbed" and stored by the operator. The recorded fmage does require approximately 80 kbytes of storage; this large size is a major deterrent to accumulating large numbers of images. On a typical run day, the accelerator is pulsed approximately 20,000 times and less than 100 inages are digitized. Our philosophy of data recording is to provide bulk recording with 1 inited resolution on the VCRs and detalled recording on a limited number of shots with the digital systems.

The video cross switch allows us to loop incoming signals through 6-bit franegrabbers, whtch are used to freeze the inage for the is between pulses. These stationary images are much easier to interpret on the fly* than the flashing inage received directly from the camera. 


\section{Digitized data analys is}

The goal of the image analysis work is to provide a spectrum of capabilities from rapid turnaround with little processing, to time-consuming detalled interactive processing of selected images to bulk p:ocessing of many images, without operator interaction.

The first 1mage processing is performed by experimentalists observing the inage. Hith a 1-Hz repetition rate. it is easy to discern systematic variations in image size, location, and intensity when accelerator parameters are changed. The ability to distribute an image to multiple monitors greatly facilitates the "instant" analys is of image data.

At the most responsive level, some data processing can take place during the 1 s between pulses. The capability exists to locate and display the centroid and "radius" of the image after each shot. Primitive algorithms must be used to obtain suffictent speed for the analysis. However, this technique of data analysis has proven very useful for studying beam shot-to-shot variation and for neasuring beam tenporal varlations when the gating of the camera is changed.

Dn the next leve1, each acquired image is transmitted from the remote ATA site to LLNL for overnight analysis. An object-finding code locates objects in the image and computes their positions and sizes as well as documenting the image with contour and histogram plots. These data are available to expertmenters the morning following the data acquisition. A typical output of the overnight analysis package is shown in Fig. 3. The code runs on a VAX computer and does not require any operator intervention.
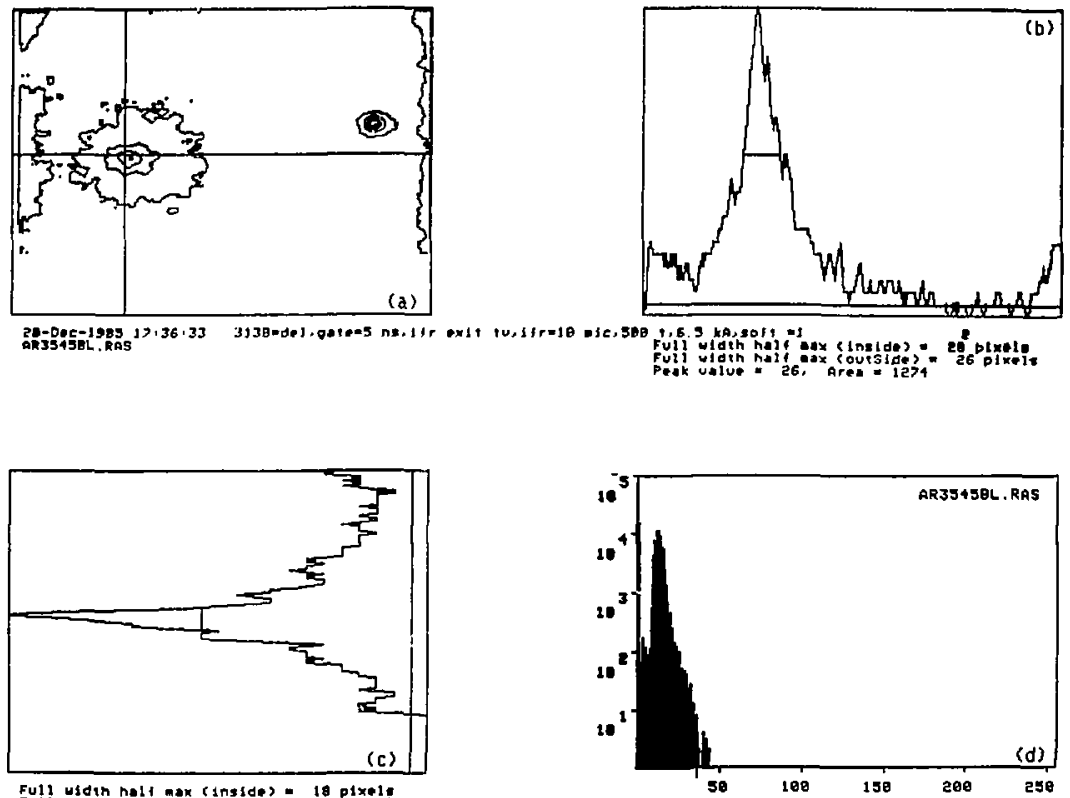

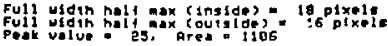

Figure 3. Automatic data analys is of a LOS image performed overnight on a VAX computer with an object-locating code.

The sample image in Flg. 3 shows data taken from the LOS documented in fig. 2 . In the upper-left corner (a) a contour plot of the light level vs $x$ and $y$ is displayed. Comparing Figs. 2 and 3 , one can observe that either the beam is far from centered on the foll or the LDS alignnent for the exit spot is off, The oval nature of the bean fmage is clearly shown. The beam radius appears to be less than a centimeter at the exit. The cross hajis on the contour plot indicate where one object was located by the image analysis code (the code did locate both bean images). Silces through the contour plot in $x$ and $y$ appear in the upper- 
rfght (b) and lower-left (c) plot positions. The histogram in the lower right (d) is valuable for determining system performance. The number of pixels is displayed on a logarithmic scale vs intensity. The highest observed amplitude is 50 , so only $20 x$ of the dynamic range is being utlized. There is a large "background" signal around 12 or 50 in intensity. The sourse of th is background is at present unknown.

For a more detailed study of a single image, we employ an interactive image manipulation code. Users can Derform various operations such as background subtraction, normalization, smoothing, row and column extraction, etc.. to obtain quantitative data from the recorded images. This is very satisfactory for analyzing single images, but more rapid and automated techniques are required for the bulk processing of data.

At the last level of image processing, a routine is maintained on the Cray computers at LLAL to perform bulk processing of image data. This code documents ea:h frame of optical data by produciny a contour plot of the image. a histogram of the pixel intensitles, and a set of silces of the inage in the $x$ and $y$ planes. This documentary output is produced on paper and fiche. Throughput is around one frame per second of conputer time, but there is 1 imited interactivity. Analysis is on a single frame only; it is up to physicists to develop the frame-to-frame correlations (e.g. radius vs time for severai shots with differing time gate locations). Because several flle transfers are required to move the data to the Cray computer network. the bulk analysis is not avallable for days to weeks after the data are acquired.

\section{Future enhancements of optical diagnostics}

To better the performance of our optical LOSs. a first step is to understand the mechanisms of light generation. Simply from a phenomenological point of view, measurements are being made to determine tf the light has the same spatial and temporal behavior as the beam current density. In a gas, the light arises frem the excitation of the molecules by collisions with beam electrons or by breakdown in the electric field associated with the bean. In some cases with well-behaved beams, the light is directly proportional to beam-current generation. When a foil is present, the light may be Cherenkov radiation or optical transition radiation (among other possiblifties). Experiments are being conducted to determine the time history. angular distribution, and polarization of light from various folls for comparison with theoretical predictions. An understanding of the light-generation mechanism may allow us to optimize the emission intensity and arigular distribution to increase the intensity of collectable light.

To enhance light-collection efficlency, we are exploring three options. A canera with extensive shielding will be placed in the tunnel to reduce the object distance by factors of several. In the past, periscopes have been used with interferometers; we are studying the advisability of using a standard periscope in conjunction with the first surface mirrors. Optical elements in the tunnel could be shielded, manually replaced, or automatically replaced. Fiber-optic bundles are being considered for image transmisston, as discussed in the companion paper. 1 However, fluorescence, transient darkening, and permanent darkening appear to be serious problems. Moreover, the relatively siall-diameter bundles are expensive, and coupling the image to the bundle is a nontrivial task.

To improve light-detection efficlency, we are deploying camera upgrades as they becone avallable. Recently. we have begun employing cameras with dual microchannel plates for low-current applications.

The data acquisition software is undergoing a major upgrade. The prinary computer for image acquisition will be a mierovax II. Thts computer has memory and speed advantages over the LSI-11s now enployed. Moreover, the microvax will reside on a network that will allow conventent transfer of experimental data to the various computers where processing occurs. Data transfer by magnetic tape will thus be eliminated. The video inserter can then be controlled by the same system that captures video inages so calibrations, etc.. can be included in the inage data file. One goal of this new system will be the display of pertinent calibraticri information with the image in real time. Another priority will be to develop acquisition systems in wich the image data are preprocessed to extract relevant ilnes or sifices for storage. This will greatly reduce the bulk of information to be stored, thereby allowing many more pulses to be recorded. Data from picture "slices" will be stored in the same format in which the "traces" from oscilloscope data are stored. Thus, much of the analysis software used for trace analys is will be usable. This tradeoff allowing data from the image to be discarded will permit the number of inages recorded and analyzed to be greatly increased.

\section{Conclusions}

Optical diagnostics will continue to play a major role in operations and experimentation at the Advanced rest iccelerator. The design and performance of the system are greatly influenced by several elements of the hostlle environment in which the system must operate. The design must also accomnodate the several very different uses made of the diagnostic information. Future expansions of the ATA optical diagnostics will be made in light of the operational nseds, realistic environment, and past experiences described in this report.

\section{Acknowledgments}

Performed jointly under the auspices of the U.S. DOE by LLNL under W-7405-EHG-4B and for the DOD under DARPh, ARPA order No. 4395, monftored by HSHC. 


\section{feferences}

1. Chong, Y. P., Cornish, J. P., and Donnelly, D., "Optical Diagnostics in the Advanced Test Accelerator (ATA) Environment." paper $787-20$ at this SPIE Canference.

2. Struve, K. H., "Radtation Induced Hoise Signals in Diagnostic Cabling on the Advanced Test Accelerator," Proc. Beams 186. Sixth International Conference on High-Power Particle Beams, Kobe, Japan, 1986. 